\title{
The effect of carbohydrate ingestion on the motor skill proficiency of s occer players
}

Clayton Zeederberg, Lloyd Leach, Estelle V. Lambert, Timothy D. Noakes, Steven C. Dennis, and John A. Hawley

This study examined the effects of ingesting a glucose-polymer (GP) solution on the motor skill proficiencies of association football (soccer) players from two teams playing during two matches in a cool environment. Fifteen minutes before each match and at halftime, players from both teams ingested $5 \mathrm{ml} / \mathrm{kg}$ of either placebo or a $6.9 \% \mathrm{GP}$ solution. GP ingestion did not improve tackling, heading, dribbling, or shooting ability. On the contrary, the mean of successful tackles was lower with GP ingestion than with placebo. The success rate for heading, dribbling, and shooting also tended to be lower in the GP than in the placebo condition. In contrast, success in passing and ball control was similar in the two conditions. Improvements in passing and ball control may have been related to a decrease in the intensity of play in the second half of the game. These data indicate that there are no measurable benefits of GP ingestion for the motor skill proficiencies of soccer players during games played in a cool environment.

Despite the vast literature on the fluid, energy, and electrolyte requirements of endurance athletes $(2,5,12,15)$,there are few well-controlled field studies on the carbohydrate (CHO) requirements of individuals who perform repeated bouts of intermittent intense exercise in team sports lasting $\leq 90 \mathrm{~min}$. Such sports, which include association football (soccer), American football, rugby, and basketball, require players to run and sprint repetitively during the course of a match.

Distances covered by soccer players during a match have been reported to range from 7 to $12 \mathrm{~km} /$ game (for review, see 4), with up to $12 \%$ of this running distance $(\sim 1.0 \mathrm{~km})$ at sprint speed $(13,16,17,21)$. In addition, players may accelerate from a stationary position 40-62 times/game (19) and change pace and/ or direction every $5 \mathrm{~s}$ (8). Such intermittent, high-intensity exercise has been reported to decrease muscle glycogen stores by $84-90 \%(6,7)$, and low muscle glycogen contents have been observed in some soccer players after only $45 \mathrm{~min}$ of play $(7,18)$. In these studies, during the second half of the matches, players with low halftime muscle glycogen stores ran slower and covered less distance than their teammates $(7,18)$.

Indirect evidence to suggest that reduced $\mathrm{CHO}$ availability may impair the highintensity exercise capacity of soccer players in the second half of a match also comes from the studies of Leatt and Jacobs (11) and Kirkendall et al. (9). Leatt and Jacobs 
(11) found that $\mathrm{CHO}$ ingestion $10 \mathrm{~min}$ before a soccer game decreased muscle glycogen utilization by $39 \%$, while Kirkendall et al. (9) showed that $\mathrm{CHO}$ ingestion increased "running distance at speed" in the second half by $30 \%$.

Improvements in soccer match play with $\mathrm{CHO}$ ingestion were also reported by Muckle (14), who assessed performances of soccer players who drank either a glucose solution or a sweetened placebo during 20 successive games played in a single season. Glucose syrup ingestion before 10 of the games prevented a $20-50 \%$ reduction in the subjects' involvement in play during the final $30 \mathrm{~min}$ of the games and increased the ratio of goals scored/goals conceded by the team, especially in the second half.

One possible explanation for the benefits of $\mathrm{CHO}$ ingestion on the goal-scoing performances of Muckle' s subjects (14) was that the players could maintain a higher exercise intensity throughout the course of a match compared to when they consumed plain water, since an adequate supply of $\mathrm{CHO}$ is required to sustain muscle power output. Alternatively, the improved performances with CHO ingestion may have been due to the players' ability to perform precise motor skills during constantly changing tactical situations during the crucial later stages of a match. A reduced blood glucose concentration might inhibit performance of skills requiring sensoryvisual information, precise postural changes, and coordinated phasic movements.

Accordingly, the aim of the current investigation was to determine the effects of $\mathrm{CHO}$ ingestion on soccer players' success in tackling opponents and in controlling, passing, dribbling, heading, and shooting the ball.

\section{Methods}

\section{Subjects}

The subjects in this study were members of two soccer teams for players under 19 years of age, playing in the Western Province Caltex Colts League in Cape Town, South Africa. This league represents the highest preprofessional level of soccer in this country and was selected because pilot studies showed that players in this league do not normally ingest $\mathrm{CHO}$ before or during training or competitive matches. Prior to the study, subjects provided written informed consent, and all procedures were approved by the Ethics and Research Committee of the Faculty of Medicine of the University of Cape Town Medical School.

\section{Field Trials}

For these investigations, the two teams played against each other on two separate occasions separated by 7 days. The teams were evenly matched in ability, finishing within one place of each other in the previous season's league competition. The two teams were also local rivals, which ensured that both matches were played in a highly competitive spirit.

Players were instructed to consume a light meal 3-4 hr before each game, similar in size and composition to that which they would normally ingest before a league game. Then, 15 min before the two matches and at halftime, prayers from both teams ingested 
$5 \mathrm{ml} / \mathrm{kg}$ body mass of either an artificially sweetened placebo or a $6.9 \% \mathrm{w} / \mathrm{v}$ short-chain glucose polymer (GP) solution containing $14.7 \mathrm{mEq} / \mathrm{L}$ sodium and $0.5 \mathrm{mEq} / \mathrm{L}$ potassium (Energade Sports Drink, Cadbury Beverages, CT). In one match, 2 defenders, 2 midfield players, and 1 forward from a team received a placebo drink, while the rest of their team consumed the GP. In the other game, the above mentioned players drank GP, while the rest of their team were given the placebo. Both matches were played in the evening (kickoff 6:30p.m.) in ambient temperatures of $13-15{ }^{\circ} \mathrm{C}$, a relative humidity of $63-69 \%$, and a wind speed of $15-22 \mathrm{~km} / \mathrm{hr}$.

Between the ingestion of the drink and the start of each match, all subjects were weighed on a Seca precision balance (Model 770, Bonn, Germany) to the nearest 0.1 $\mathrm{kg}$ so that whole-body sweat loss could be estimated from the decline in body mass measured at the end of the match, plus the volume of fluid ingested, minus the volume of any urine passed. Then, 3 defenders, 2 midfield players, and iforward were fitted with Sports Tester PE300o shortwave telemetry heart rate monitors (Polar Electro, Kempele, Finland) to record heart rates (HRs) every $15 \mathrm{~s}$ throughout the match. Those data were used to indicate any effects of CHO ingestion on the relative work rates of these players during the two games.

The players' skill proficiency in tackling opponents and in controlling, passing, dribbling, heading, and shooting the ball was recorded with two video cameras placed diagonally opposite each other on each side of the two halves of the playing field. Videotapes were subsequently edited and spliced so that there was a continuous close view of the ball-play in each half of the field; this tape was used to evaluate the players' soccer motor skills (described later).

Within $60 \mathrm{~s}$ of the end of a match, blood samples (10ml) were drawn from an antecubital vein and placed in ice-cold tubes containing potassium oxalate and sodium fluoride. Back in the laboratory, the tubes were centrifuged at $3,000 \mathrm{rev} / \mathrm{min}$ for $10 \mathrm{~min}$ at $4{ }^{\circ} \mathrm{C}$, and the supernatant was removed and stored at $-20{ }^{\circ} \mathrm{C}$ for subsequent analyses of plasma glucose and lactate concentrations. Plasma glucose concentrations were measured in duplicate with an automated glucose analyzer (LM3 Glucose Analyzer, Analox Instruments, London, U.K.), and plasma lactate concentrations were determined with a standard spectrophotometric enzymatic assay (Bergmeyer, Germany).

\section{Evaluation of Soccer Motor Skill Proficiency}

Proficiency in tackling opponents and in controlling, passing, dribbling, heading, and shooting the ball was first evaluated in a pilot study. In that study, 1ophysical education students watched a videotape of a single player from a previously recorded soccer match and scored the player's performance of soccer skills as either unsuccessful or successful. Success criteria are listed below, together with the coefficients of variation (CV) of the 10 evaluations of each skill:

- Tackling: A player tackles an opponent to obtain possession of the ball or defensively kicks it out of play ( $\mathrm{CV}=4.5 \%)$. 
- Controlling: A player receives a pass and is able to pass the ball to a member of his team or diibble it or shoot it at the opponent's goal ( $\mathrm{CV}=4.9 \%)$. Passing: A player kicks the ball to a teammate without interception by the opposition or over the sideline for a defensive clearance ( $\mathrm{CV}=5 \%)$.

- Dribbling: A player passes an opponent while remaining in possession of the ball $(\mathrm{CV}=4.5 \%)$.

- Heading: A player heads the ball to a teammate who is able to retain possession, toward the opponent's goal, or to safety off-field ( $\mathrm{CV}=\mathrm{o} \%)$. Shooting: A player scores a goal or forces the goalkeeper or a defender to clear the ball to prevent a goal $(\mathrm{CV}=0 \%)$.

The same criteria were used in the present study to evaluate the effects of $\mathrm{CHO}$ ingestion on the players' percentages of success in tackling, controlling the ball, passing, dribbling, heading, and shooting. For these evaluations, the same Io physical education students assessed the soccer motor skill performances of 2 of the 20 outfield players from the two teams over the two matches. Each student was assigned 1 player from each team and was not informed as to when the players ingested $\mathrm{CHO}$ or placebo.

\section{Statistical Analyses}

All data are expressed as means \pm standard deviations (SD). A two-way analysis of variance for repeated measures was used to analyze differences in response to treatment from the first to the second half.

\section{Results}

In spite of the cool, somewhat windy conditions for the two matches, the players' sweat losses in each game averaged 1.0 $\pm 0.2 \mathrm{~L}$ and were similar in the placebo and GP groups.

The players' postgame plasma glucose and lactate concentrations were also unaffected by GP or placebo ingestion. Plasma glucose concentrations in the placebo and GP groups were $5.1 \pm 0.9$ and $5.3 \pm 0.7 \mathrm{mmol} / \mathrm{L}$, respectively, and plasma lactate concentrations were $4.7 \pm 2.1$ and $4.5 \pm 2.4 \mathrm{mmol} / \mathrm{L}$, respectively.

GP ingestion also had no effect on the 20 outfield players' success in passing and controlling the ball (Figure $\mathbf{I}$, upper panels). In the placebo and GP groups, success in passing tended to rise from a range of $61-63 \%$ in the first half to a range of $68-69 \%$ in the second half of the game $(p<.06)$, while success in ball control tended to increase from between $72-73 \%$ to $76-81 \%$ over the same periods $(\mathrm{p}<.09)$.

There was also no benefit of GP ingestion on success in tackling, heading, dribbling, and shooting (Figure l, lower panels). On the contrary, the overall successes in tackling and in heading, dribbling, and shooting the ball tended to be slightly lower in the GP than in the placebo conditions, but neither difference was significant. With GP ingestion, successes in tackling were reduced from $63 \pm 8 \%$ to $53 \pm 12 \%$, respectively, and successes in heading, dribbling, and shooting were reduced from $43 \pm 6 \%$ to $37 \pm$ $7 \%$, but neither difference was significant. 
The numbers of tackles, headers, dribbles, and shots in the GP group also tended to be Jess than in the placebo group in second half of the game (Figure 1), but this difference was not significant and was not associated with any differences in HR (Figure 2). With or without GP ingestion, HR dropped significantly from -165 beats/min for the first hour of the match to -150 beats/min during the final $25 \mathrm{~min}$ of the game (Figure $2 ; p$ $<.05)$.
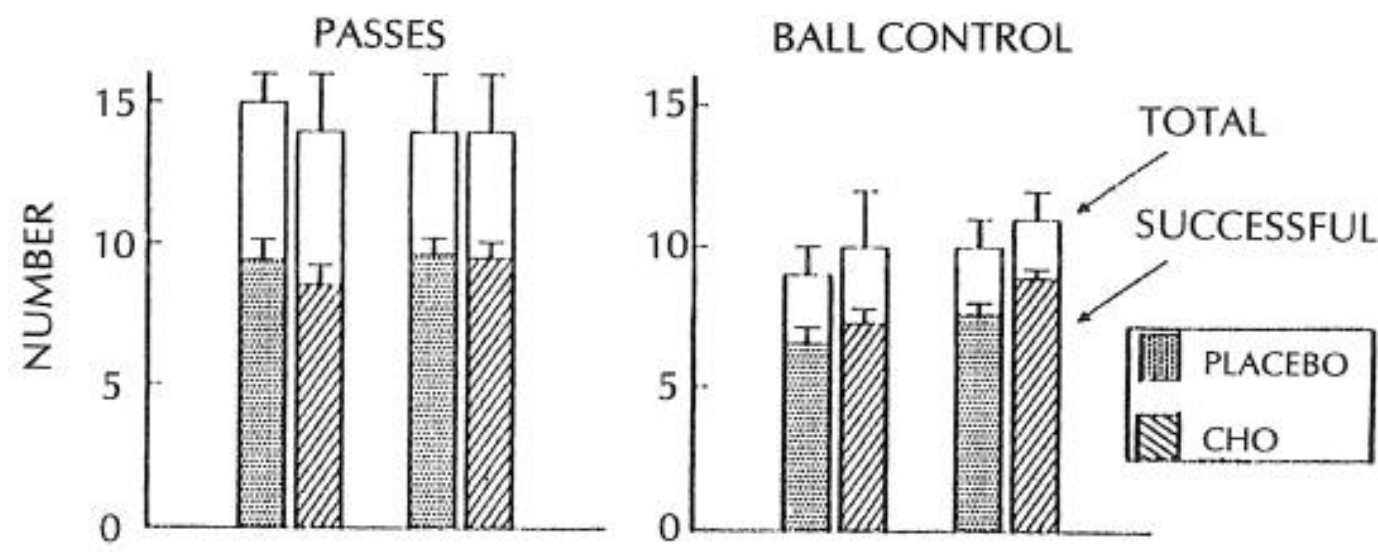

TACKLES
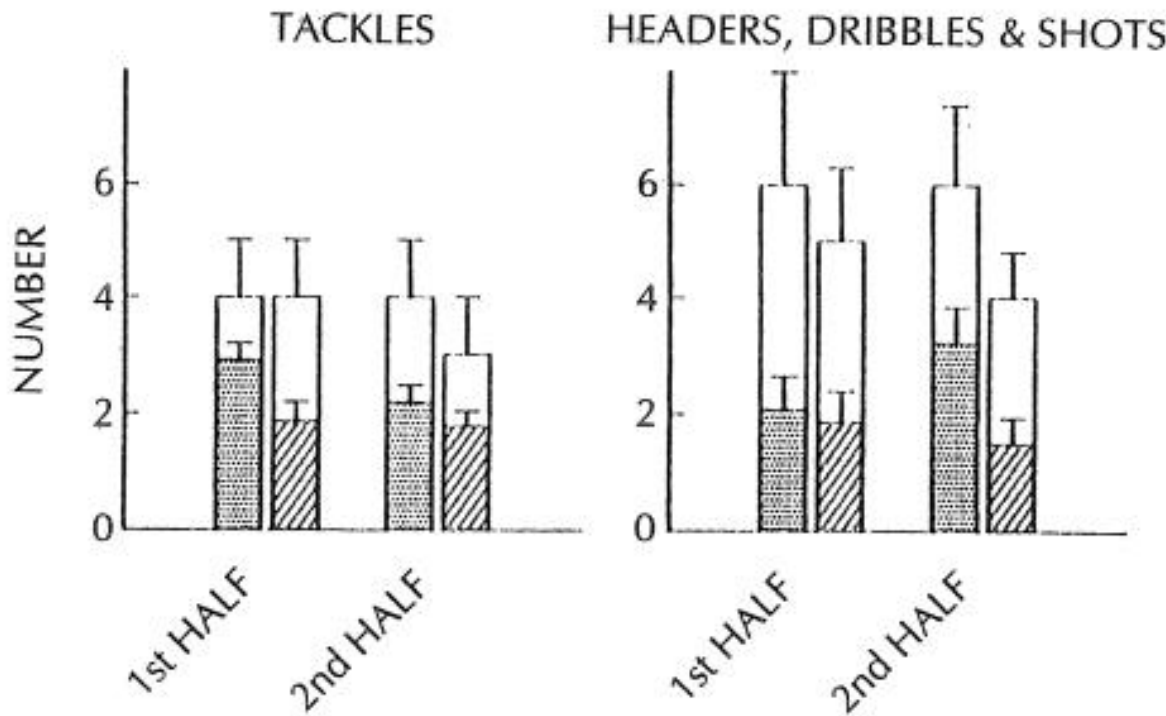

Figure 1- The effect of ingesting either a glucose-polymer solution or placebo on the motor skill proficiency of soccer players. The top panel indicates the number of successful passes and ball controls each half, while the bottom panel shows the number of successful tackles, headers, dribbles, and shots.

\section{Discussion}

There is a lack of well-controlled field studies examining the possible ergogenic effect of $\mathrm{CHO}$ ingestion on performances during team sports. When a crossover design is employed, it is difficult to adequately control environmental conditions, tactics, and motivational factors between trials or matches. Maughan (12) noted that many of the early studies purporting to show a beneficial effect of the ingestion of CHO-electrolyte solutions in sports such as soccer were so poorly controlled that the results are of little or no value. In this study we have attempted to overcome such criticisms as follows: Players on both teams were the same for both matches, the environmental and playing 
conditions (e.g., pitch) were constant, and the experiment was a single-blind, crossover design.

With this experimental design we found that the ingestion of a CHO- electrolyte supplement before and during $90 \mathrm{~min}$ of soccer match play had no significant effect on the motor skill proficiency of outfield soccer players (Figure 1).

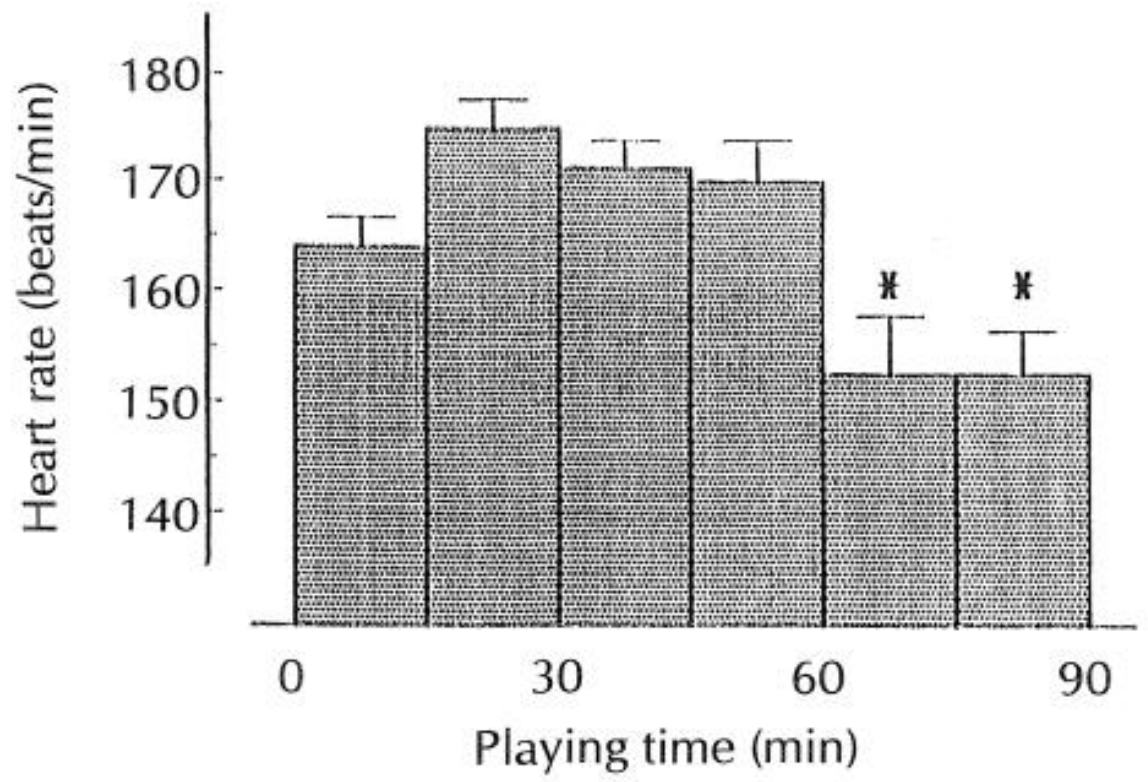

Figure 2- The means and standard deviations of the heart rates recorded for 6players during the two matches (pooled data)

There was no benefit of $\mathrm{CHO}$ ingestion on the players' capacity to pass or control the ball successfully (Figure 1, upper panels) or on their ability to tackle, head, dribble, or shoot more accurately (Figure I, lower panels).

A possible explanation for the players' similar responses to the two experimental conditions may be that in no instance was there any evidence of postmatch hypoglycemia. Plasma glucose concentrations were maintained at $>5 \mathrm{mmol} / \mathrm{L}$, regardless of whether players ingested $\mathrm{CHO}$. This finding is in accord with that of Leatt (10), who reported plasma glucose concentrations of $4.7 \mathrm{mmol} / \mathrm{L}$ after a Canadian league division two match. However, our finding is in contrast to that of Ekblom (3), who found average plasma glucose concentrations of $3.8 \mathrm{mmol} / \mathrm{L}$ immediately after a Swedish first division match, with several of the players in his study presenting with frank hypoglycemia (i.e., plasma glucose concentration $<3.0 \mathrm{mmol} / \mathrm{L}$ ) after $90 \mathrm{~min}$ of play.

Although there was no evidence of hypoglycemia in any of the subjects under investigation, and plasma lactate concentrations were moderately high $(-5 \mathrm{mmol} / \mathrm{L})$ and in agreement with other studies (1), the average heart rates of the outfield players during the last quarter of the matches were significantly lower than during the first hour (Figure 2). Although muscle glycogen content was not measured in the current investigation, other studies $(6,7)$ have suggested that a slowing of the pace of the game is associated with muscle glycogen depletion. Another study found that at the 
end of a game, players with low muscle glycogen levels covered less distance, walked more, and sprinted less than players with higher muscle glycogen levels (18).

Associated with the drop in work rate during the final period of the games was a concomitant increase in the players' success in passing and controlling the ball (Figure 1, upper panel), regardless of whether they ingested placebo or GP. This finding indicates that as a player's overall work rate drops during the later stages of a match, there is a compensatory increase in the number of successful passes, which probably results in a team maintaining possession of the ball longer and thus having to run less.

Finally, despite the ingestion of a total of $-700 \mathrm{ml}$ of fluid immediately before and during the games, and also despite the cool environmental conditions, fluid losses in the outfield players averaged $1 . \mathrm{O} \mathrm{L}$, with some players losing up to $1.5 \mathrm{~L}$ in a game. As a fluid loss of only $1.8 \% \mathrm{BM}$ (or $1.2 \mathrm{~L}$ ) has been shown to impair performance in the heat (20), and as the rules of soccer currently permit the ingestion of fluids only during halftime (i.e., after $45 \mathrm{~min}$ of play), it seems likely that teams playing in hot environments should be afforded the opportunity to drink more frequently, as has been recently proposed (4). However, whether CHO supplementation would improve motor ability and/or energetics of soccer match play in a hot climate remains to be established. All we can conclude from the current study is that in cool environmental condition s, the ingestion of a CHO-electrolyte solution had no measurable effect on the motor skill proficiency of soccer players. 


\section{References}

1. Bangsbo, J. The physiology of soccer-with special reference to intense intermittent exercise. Acta Physiol. Scand. Suppl. 619, 1994.

2. Coyle, E.F., and M.H. Hamilton. Fluid replacement during exercise: Effects or pysiological homeostasis and performance. In Perspectives in Exercise Science anc $S$ orts M edicine: Vol. 3. Fluid Homeostasis During Exercise, C.V. Gisolfi and D.R La mb (Eds.). Carmel, IN: Brown \& Benchmark, 1990, pp. 281-308.

3. Ekblom, B. Applied physiology of soccer. Sports Med. 3 50-60, 1986.

4. Hawley, J.A., Dennis, S.C., and Noakes, T.D. Carbohydrate fluid and electrolyte requirements of the soccer player: A review. Int. J. Sport - Nutr. 4:221-236, 1994.

5. Hawley, J.A., Dennis, S.C., and Noakes T.D. Carboh drate, fluid, and le tr lyte requirements during prolonged exercise. In Sports Nutrition: Minerals and Electrolytes, C.V. Kies and J.A. Driskell (Eds.). Boca Raton, FL: CRC Press, 1995, pp. 235 - 265 .

6. Jacobs, I.,N. Westlin, M. Ramusson, and B. Houghton. Muscle glycogen and soccer players. Eur. J. Appl. Physiol. 48:297-302, 1982.

7. Karlsson, H.G. Kolhydratomsattning under enfobollsm tel [Carbohydrat during a footbal 1 match] (Report,Department of Physiolog I ). Stockholm:Institute, I 969.

8. Kirkendall, D.T. The applied sport science of soccer. Phy $S$ ortsmed. 13:5_-5

9. Kirkendall, D.T.,C. Foster, J.A. Dean, J. Gorgan, and N .N. T mpson. Effect of polymer supplementation on performance of soccer playe1 . I Science and Football. T. Reilly (Ed.). London: Spon, 1988.

10. Leatt, P.B. The effect of glucose polymer ingestion on skele al 1uscle glycoge $1 \mathrm{~d}$ during soccer match play and its resynthesis following $a w h$. Unpublished thesis, University of Toronto, Toronto, ON, 1986.

11. Leatt, P.B., and I. Jacobs. Effect of glucose polymer ingestion on glycoger during a soccer match. Can. J. Sport Sci. 14: 112-116, 199.

12. Maughan, RJ. Carbohydrate-electrolyte solutions during prolonged exercise. In Perspectives in Exercise Science and Sports Medicine: Vol 4. Performance in Exercise and Sport, D.R. Lamb and M.H. Brown \& Benchmark, 1991, pp. 35-85.

13. Mayhew, S.R., and H.A. Wenger. Time,.-motion analysis of professional soccer. $J$. Hum. Mov. Stud. 11:49-52, 1985.

14. Muckle, D.S. Glucose syrup ingestion and team performance in soccer. $\mathrm{Br} . \mathrm{J}$. Sports Med. 7:340-343, 1973.

15. Noakes, T.D. Fluid replacement during exercise. In Exercise and Sport Sciences Reviews (Vol. 21), J.o. Holloszy (Ed.). Baltimore:Williams \&Wilkins, 1993, pp. 297330.

16. Reilly, T., and V. Thomas. A motion analysis of work rate in different positional roles in professional football match play. J. Hum. Mov.Stud. 2:87-97, 1976.

17. Reilly, T., and V. Thomas. Estimated daily energy expenditures of professional association footballers. Ergonomics 22:541-548, 1979.

18. Saltin, B. Metabolic fundamentals in exercise. Med.Sci.Sports Exerc.5:137-146, 1973 .

19. Smodlaka, V.N. Cardiovascular aspects of soccer. Phys. Sportsmed. 6:66-70, 1978. 20. Walsh, R.M., T.D. Noakes, J.A. Hawley, and S.C. Dennis. Impaired highintensity cycling performance time at low levels of dehydration. Int. J. Sports $M$ ed. 15:392-398, 1994. 
21. Withers, R.T., Z. Maricic, S. Wsilewski , and L. Kelly. Match analysis of Australian professional soccer players. J. Hum. Mov. Stud. 8:159-176, 1982.

\section{Acknowledgments}

This study was supported by a grant from Bromar Foods, Salt River, Cape Town. 